\title{
Measures and dynamics of entanglement
}

\author{
A. Buchleitner ${ }^{1}$, A.R.R. Carvalho ${ }^{2}$, and F. Mintert ${ }^{3}$ \\ 1 Max-Planck-Institut für Physik Komplexer Systeme, Nöthnitzer Strasse 38, 01187 Dresden, Germany \\ 2 Dept. of Physics, Faculty of Science, Australian National University, ACT 0200, Australia \\ 3 Physics Department, Harvard University, Cambridge, Massachusetts 02138, USA
}

\begin{abstract}
We briefly review our recent work on how to quantify and how to measure the entanglement inscribed into quantum states which evolve under incoherent environment coupling.
\end{abstract}

\section{Introduction}

Quantum entanglement is considered to be a central resource of quantum information processing: entanglement is a key ingredient in quantum teleportation [1], and is used to perform conditional two-qubit operation, which are primordial for running a quantum algorithm [2]. There is a large body of literature on the abstract, rather mathematical theory of entanglement [3] - of pure as well as of mixed states -, and equally so can one find a considerable number of proposals on manipulating the entanglement of two qubits [4] - the smallest quantum systems which can exhibit quantum correlations. On the experimental side, single and two-qubit operations are by now very well controlled [5], and the important challenges arise with the control and characterization of the entanglement inscribed in quantum systems with an ever larger number of constituents [6-8]: to outperform a classical supercomputer, also an all-purpose quantum computer has to run on large quantum registers, i.e., several hundred or thousand rather than two qubits. Yet, very little is known on the time evolution of entanglement under realistic conditions, in a multicomponent quantum system of increasing size.

Indeed, if we take it as granted that entanglement is a central resource, some kind of special "fuel" on which a quantum computing engine runs, then the central question for the experimentalist is the time scale on which this fuel is exhausted, as compared to the time scale needed to perform the actual algorithmic task. Since entanglement is a manifestation of coherent superpositions of many-particle eigenstates of a quantum register, it is clear that decoherence will be detrimental for quantum entanglement, and a quantitative theory to assess entanglement decay rates under environment coupling is very much in need. Since, in addition, the spectral density of a composite quantum system increases with its number of constituents, it is also clear that a multicomponent system will respond differently to environmental noise than a two-qubit system, and very general arguments suggest that the larger the system the faster the decay of multiparticle coherences $[9,10]$, and thus of entanglement. Therefore, if we take the perspective of a quantum computer seriously, then the theoretical challenge ahead is to develop a theory which focusses on the scaling properties of entanglement, and notably of its robustness against environment coupling, with increasing systems size [11,12]. Since the Hilbert space dimension of a composite quantum system grows exponentially both with its number of constituents as well as with the dimension of the individual factor spaces, this is a highly nontrivial challenge, both for experiment and theory: on the theoretical side, one needs to find computationally efficient tools for the characterization of entanglement, such as to 


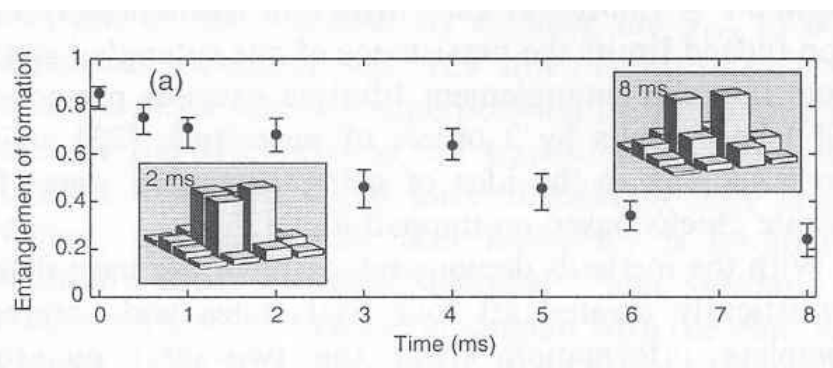

Fig. 1. Time evolution of the entanglement of a maximally entangled two-qubit state under environment coupling [20]. The insets show the statistical operator representing the quantum state at different times. While entanglement decays monotonously, the coherences of the density matrix are redistributed, but not clearly damped out. This highlights the nonlinear dependence of the state's entanglement on the statistical operator (Courtesy of C. Roos).

inspire experimental strategies to identify specific classes of non-classical correlations inscribed in arbitrary quantum states of increasing dimension - without requiring a complete knowledge of the state [13-16]. Thus, the theoretical focus on the scaling properties of entanglement is intimately related to the experimental need for efficient ways of entanglement measurement.

Finally, we should be aware of the fact that the desire to run a large scale quantum computer implies that we aim at exploring quantum interference effects on a macroscopic, or, at least, mesoscopic scale. The fact that coherent superpositions of macroscopic objects in the world around us are scarce, provides another very explicit hint to the problems yet to overcome. Necessarily, large composite systems are always in some sense open systems, i.e., they are coupled to uncontrolled/unobserved degrees of freedom, what implies decoherence [17]. In this sense, the possibility of building a real quantum computer is conditioned on our ability to avoid the quantum-classical transition on macroscopic scales, and this immediately clarifies why experiments on the controlled creation of many-particle entanglement [6-8] and on the coherence properties of heavy particles represent just two faces of the same medal [18].

\section{Quantifying entanglement}

Before we can address the dynamical evolution of entanglement, we need efficient tools to distinguish separable from entangled states, and to quantify the amount of entanglement inscribed into a given state. This problem has a complete solution for the simplest possible case of two qubits - i.e., for systems living on a four dimensional tensor Hilbert space $H=H_{1} \otimes H_{2}$ composed of two two-dimensional Hilbert spaces -, and mixed state entanglement can be derived purely algebraically from the density matrix of such $2 \times 2$ system [19]. Indeed, first experiments did monitor the time evolution of the entanglement of an initially maximally entangled $2 \times 2$ state under environment coupling, through direct tomography of the density matrix at different times $t[20-22]$. The insets in Fig. 1 show the redistribution of the coherences of the density matrix as time evolves, together with the monotonous decrease of entanglement [20]. Remarkably, however, it is not obvious from the density matrix' time evolution that the coherences really decay - while entanglement does: this is an immediate manifestation of the nonlinear dependence of entanglement on the density operator, which is at the very heart of the entanglement characterization problem, and which turns into a truly hard problem for systems of larger dimension, where tomography no more provides a realistic strategy for state analysis, simply due to the exponential increase of the required experimental resources.

The matter thus becomes much more involved when we increase the number of subsystems or the subdimension of the factor spaces $H_{i}$. However, it is this latter problem which we have to tackle if we want to talk about large scale quantum computing! Therefore, let's look a bit deeper into this subject. 


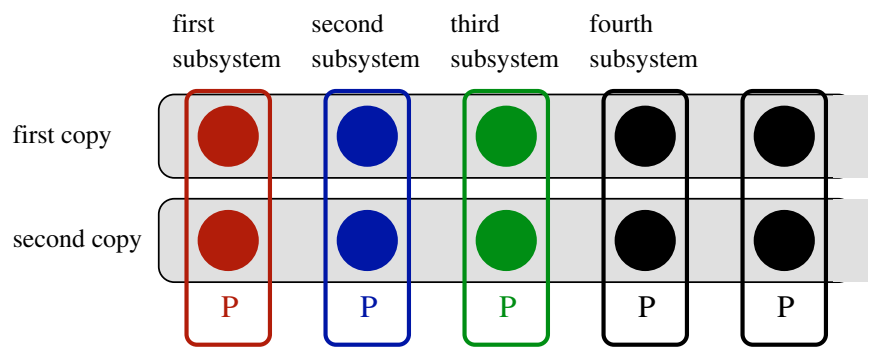

Fig. 2. Schematic representation of the two-copy scheme for a multipartite system. The self-adjoint operator $A$ is composed of products of symmetric and antisymmetric projectors $P$ on the (anti)symmetric subspaces of the first and second copy of either one of the subsystems $1 \ldots N$, with the constraint that $A$ be symmetric under the simultaneous exchange of the copies of all subsystems [26]. For $N=2, A$ is given by the product of two antisymmetric operators: $A \propto P_{-}^{(1)} \otimes P_{-}^{(2)}$.

\subsection{Entanglement measures}

A pure state $|\Psi\rangle$ on a bipartite Hilbert space $H=H_{1} \otimes H_{2}$ is called separable if it can be written as a product $|\Psi\rangle=|\phi\rangle \otimes|\eta\rangle$ of any two vectors $|\phi\rangle \in H_{1}$ and $|\eta\rangle \in H_{2}$; otherwise, the state is entangled. Possible measures of pure state entanglement are [23] provided, e.g., by the von Neumann entropy of the reduced density matrix of either one of the subsystems, or by concurrence. They have a nice interpretation in terms of the information loss induced by tracing out one of the subsystems, and concurrence is defined as

$$
c(\Psi)=\sqrt{2\left(1-\operatorname{tr} \rho_{r}^{2}\right)},
$$

in terms of the reduced density matrix $\rho_{r}$ of one of the subparties. In particular, this definition vanishes precisely for separable states, and is immediately amenable to bipartite systems of arbitrary finite dimension [24]. In the following, we will use concurrence as our preferred entanglement measure, essentially since its definition allows for algebraic manipulations which would be much harder, e.g., for the von Neumann entropy.

It may appear suggestive to generalize concurrence for mixed states

$$
\rho=\sum_{j} p_{j}\left|\Psi_{j}\right\rangle\left\langle\Psi_{j}\right|
$$

as the weighted average of the pure state concurrences of its pure state components. However, since the pure state decomposition of mixed states is not unique, this is not a viable strategy. Rather, one has to take the infimum over all possible pure state decompositions [25],

$$
c(\rho)=\inf _{\left\{p_{j}, \Psi_{j}\right\}} \sum_{j} p_{j} c\left(\Psi_{j}\right)
$$

which has an explicit solution in the $2 \times 2$ case [19], but in general defines an optimization problem of rapidly increasing dimension as the system dimension increases. Furthermore, a numerical solution of the optimization problem will always yield upper bounds for concurrence, what cannot help to distinguish separable from entangled states: what is needed are lower bounds.

These can be derived once one realizes that pure state concurrence can be reformulated as

$$
c(\Psi)=\sqrt{\langle\Psi|\otimes\langle\Psi|A| \Psi\rangle \otimes| \Psi\rangle},
$$

with a self-adjoint operator $A$ acting on two copies of the state to be analyzed [26], see Fig. 2 . $A \propto P_{-}^{(1)} \otimes P_{-}^{(2)}$, with $P_{-}^{(1,2)}$ the projectors on the antisymmetric subspaces of the space of the first and second copy of subsystems 1 or 2 . One easily verifies that, also in this formulation, $c(\Psi)$ vanishes exactly for separable states. 


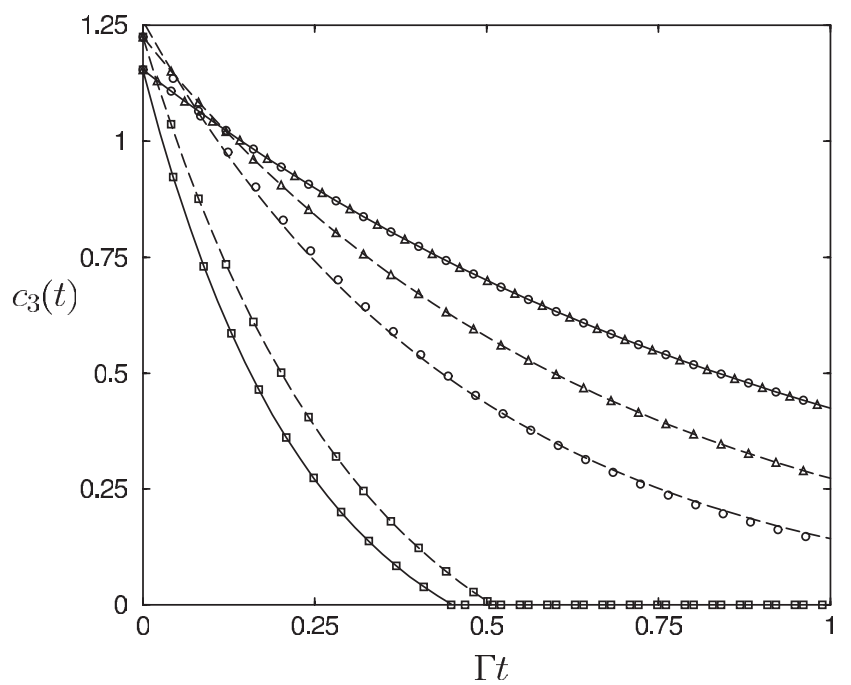

Fig. 3. Decay of the three-partite concurrence $c_{3}[26,28]$ of three-partite W (solid lines) and GHZ (dashed lines) states [10], where each register qubit is individually coupled (with strength $\Gamma$ ) to its private zero temperature (circles), infinite temperature (squares), or dephasing (triangles) environment - therefore, the qubits cannot interact through the environment. Concurrence was here calculated by use of the quasipure approximation [30], which is the computationally "cheapest" entanglement quantifier of our hierarchy of lower bounds. However, the accuracy of the approximation was found to be excellent by direct comparison to optimal lower and upper bounds of concurrence at different times.

Indeed, the algebraic structure of (4) lends itself for an immediate generalization for multipartite systems, where $A$ is composed of products of symmetric and antisymmetric projectors with the constraint that it be symmetric under exchange of the copies of all subsystems, since $|\Psi\rangle \otimes|\Psi\rangle$ is symmetric under this operation (see Fig. 2). Finally, mixed state concurrence of a general multipartite mixed state $\rho$ is given by

$$
c(\rho)=\inf _{\left\{p_{j}, \Psi_{j}\right\}} \sum_{j} p_{j} \sqrt{\left\langle\Psi_{j}\left|\otimes\left\langle\Psi_{j}|A| \Psi_{j}\right\rangle \otimes\right| \Psi_{j}\right\rangle},
$$

where $A$ needs further specification for the specific type of multipartite correlation to be addressed $[27,26]$. Once again, $c(\rho)$ vanishes exactly for completely separable multipartite states, for any specific definition of $A$ under the above symmetric constraint. Whether this general definition of $c(\rho)$ also defines an entanglement monotone is a much more intricate question, which is addressed in detail in [28]. Though, all multipartite concurrences which we shall quantitatively evaluate in the sequel of the present review indeed are entanglement monotones [28].

Equation (5) allows for the derivation of a hierarchy of lower bounds of mixed state concurrence of multipartite quantum systems of arbitrary finite dimension, which are obtained by optimization over a considerably reduced optimization space as compared to eq. $(3)[29,26]$, or even by simply diagonalizing a matrix of the same dimension as $\rho$ [30]. Since the general definitions of the relevant algebraic quantities are rather involved, the interested reader is refered to the original papers $[29,26,30]$ for details. This hierarchy helps quite a bit in reducing the computational effort for efficient entanglement characterization, and allowed us to address, e.g., the robustness of the entanglement (quantified by its decay rate) of maximally entangled bipartite [31], W, GHZ [10], or elliptic island states [32] in quantum systems encoded in quantum registers of increasing size $N$, the time evolution of bipartite entanglement generated by random Hamiltonians [30], and equally so the performance of entanglement creation schemes [26], under incoherent coupling to public or private baths. As an example, Fig. 3 shows the decay of the entanglement of three-partite $|\mathrm{W}\rangle=(|001\rangle+|010\rangle+|100\rangle) / \sqrt{3}$ and $|\mathrm{GHZ}\rangle=(|000\rangle+|111\rangle) / \sqrt{2}$ states, when each qubit is coupled to a private bath (i.e., the qubits cannot interact through the environment) with coupling strength $\Gamma[10]$. 

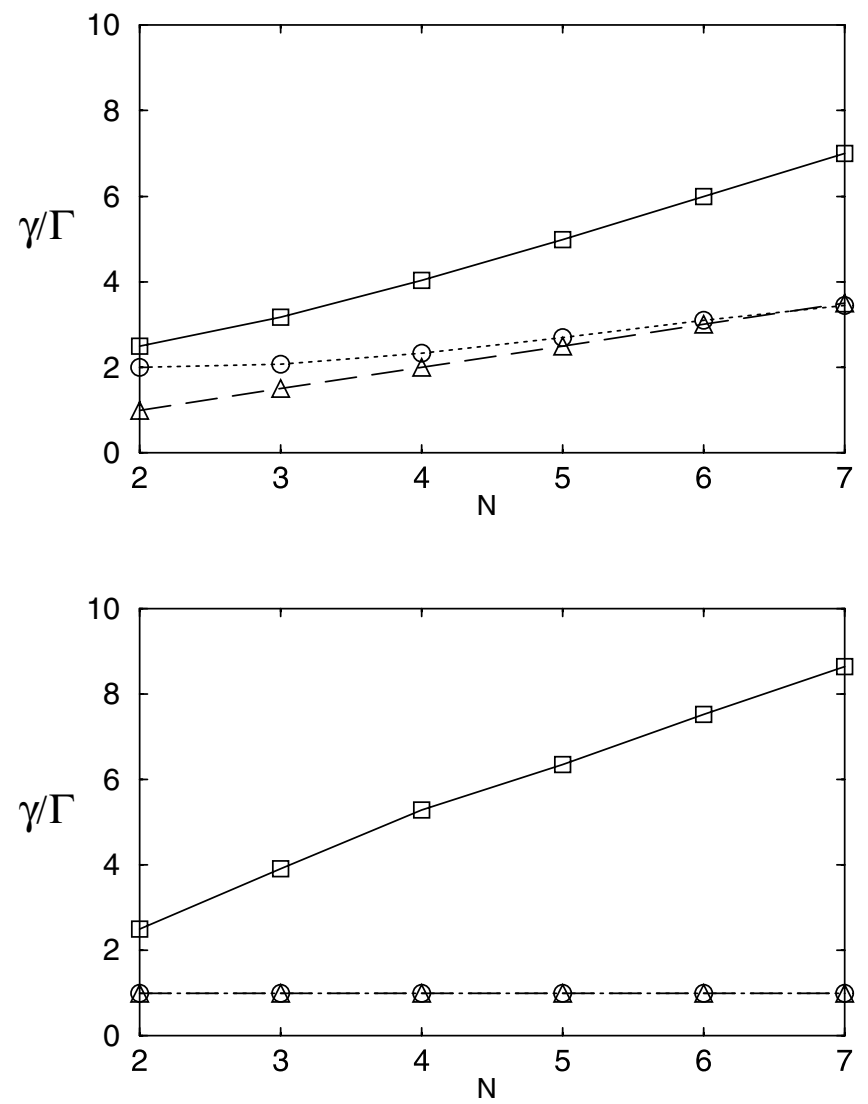

Fig. 4. Scaling of the entanglement decay rate $\gamma$ of GHZ (top) and W (bottom) states with the register size $N$, for a zero temperature (circles), infinite temperature (squares), and dephasing (triangles) environment [10]. As to be expected, $\gamma$ in general increases with $N$, except for W states coupled to zero temperature and dephasing environments, where $\gamma$ is independent on $N$. This is essentially due to the fact that W states bear only one excitation, independently of $N . \gamma$ can be derived analytically for GHZ states under dephasing, and for W states under zero temperature environment coupling [10,31], what once again allows for an independent verification of our lower entanglement bound by quasipure approximation.

Different decoherence processes - spontaneous emission, noise, and dephasing - were modeled with the standard master equation formalism (see eq. (7) hereafter, with suitably chosen operators $J_{k}$ ), and the N-partite concurrence $[10,26]$

$$
c_{N}(\Psi)=2^{1-\frac{N}{2}} \sqrt{\left(2^{N}-2\right)\langle\Psi \mid \Psi\rangle^{2}-\sum_{j} \operatorname{tr} \rho_{j}^{2}},
$$

was used as an entanglement measure (here for $N=3$ ) [26,28], where the sum over $j$ runs over all nontrivial reduced density matrices $\rho_{j}$ deduced from $\Psi$. For all cases, we can extract a typical decay rate $\gamma$, at least for short times, as illustrated in Fig. 3. The same can be done for $\mathrm{W}$ and GHZ states on larger $N$-qubit quantum registers, and Fig. 4 shows the dependence of the decay rates on the register size $N$.

As to be expected, $\gamma$ grows with $N$ in most cases, what highlights the difficulty to construct a large scale quantum computer. However, we also observe that $\mathrm{W}$ states under dephasing and spontaneous emission exhibit entanglement decay rates independent of $N$, what identifies these states as somewhat more robust under the scalability requirement. 


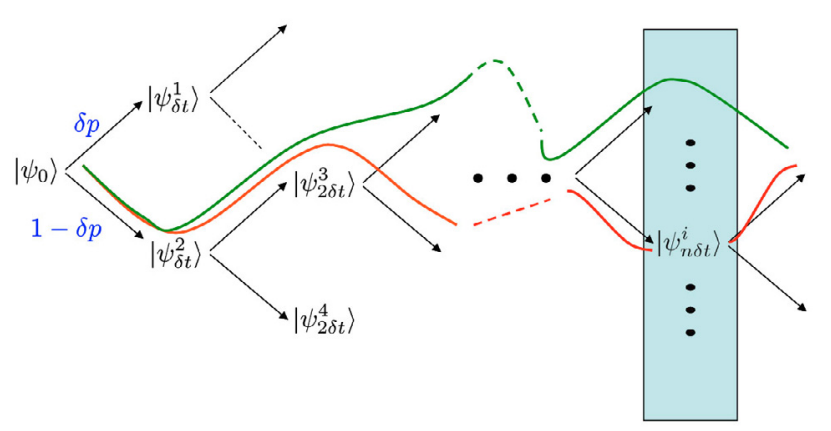

Fig. 5. Schematic representation of the time evolution of different quantum jump trajectories in the unraveling approach. At each time step, a quantum jump occurs with probability $\delta p$, and no event is observed with probability $1-\delta p$. An ensemble of quantum trajectories provides an ensemble of pure states $\left|\psi_{n \delta t}^{i}\right\rangle$ at time $t=n \delta t$, which is a valid decomposition of the density matrix at that time.

\subsection{Entanglement dynamics revisited}

With the above, we can quantify entanglement dynamics in arbitrary finite dimensional quantum systems, but still need to rely on the time evolution of the density matrix $\rho(t)$ itself: at each time $t$, we apply the above prescription $(5,6)$ to deduce the state's entanglement from $\rho(t)$. Instead, we would like to develop a scheme for the direct monitoring of entanglement evolution in real time. As we will show in the present section, this can be achieved by unraveling [33] entanglement in a quantum trajectory treatment.

To set the scene, let us remember that the wide-spread description of incoherent state evolution by a master equation of the type

$$
\frac{d \rho}{d t}=-\frac{i}{\hbar}\left[H_{\mathrm{sys}}, \rho\right]+\sum_{k} \frac{1}{2}\left(2 J_{k} \rho J_{k}^{\dagger}-J_{k}^{\dagger} J_{k} \rho-\rho J_{k}^{\dagger} J_{k}\right)
$$

- which we used to generate the results of Figs. 3 and 4 - can be substituted by a stochastic pure state evolution of the initially pure state $\left|\Psi_{0}\right\rangle$, mediated by the quantum jump operators $J_{k}$ and a non-Hermitian, free evolution generated by $H_{\text {eff }}=H_{\text {sys }}-i \hbar \sum_{k} J_{k}^{\dagger} J_{k} / 2$ [33]. A quantum jump occurs under the action of $J_{k}$ on $|\Psi(t)\rangle$, with probability $\delta p_{k}$, and is associated with the detection of a specific event, e.g., the emission of a spontaneous photon. If no event is detected, with probability $1-\sum_{k} \delta p_{k},|\Psi(t)\rangle$ evolves under the action of $H_{\text {eff }}$, always remaining in a pure state. Since the occurence of a given event is probabilistic, a single pure state trajectory evolves stochastically, and the state $\rho(t)$ generated by the master equation is recovered after lumping together the stochastically evolved states $|\Psi(t)\rangle$, for different realizations of the stochastic jump process, as illustrated in Fig. 5. Thus, the quantum jump approach immediately yields a pure state decomposition of $\rho(t)$, for arbitrary $t$, which is completely determined by the detection record of the quantum jumps. Since pure state concurrence of the individual pure states $|\Psi(t)\rangle$ is easily evaluated, at least in the bipartite case, the detection record amounts to a direct monitoring of entanglement evolution under incoherent dynamics. The average pure state concurrence after a first time step $\delta t$ reads:

$$
\bar{c}(\delta t)=\left(1-\sum_{k=1}^{N} \delta p_{k}\right) c\left(\Psi_{\delta t}^{N+1}\right)+\sum_{k=1}^{N} \delta p_{k} c\left(\Psi_{\delta t}^{k}\right) .
$$

But ... what about the infimum in eq. (3)? Is the pure state decomposition of $\rho(t)$ obtained by the stochastic pure state evolution optimal in that sense? Of course, in general, it isn't, but we can explore the invariance of the master equation (7) under the following transformation of the jump operators:

$$
L_{k, \pm}=\frac{\mu_{k} \operatorname{Id} \pm \sum_{i} U_{k i} J_{i}}{\sqrt{2}}
$$




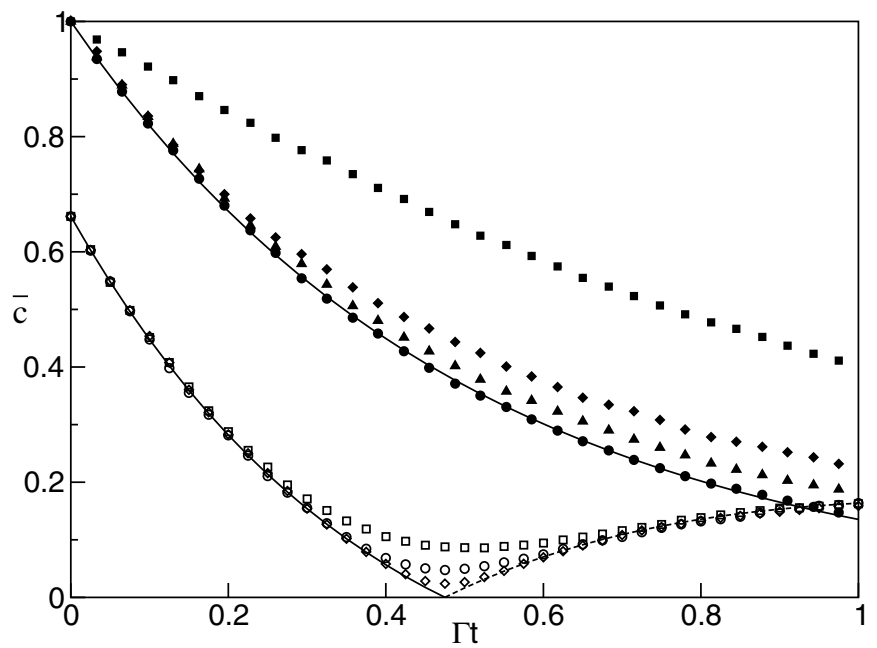

Fig. 6. Time evolution of the bipartite mixed state concurrence for initial states $\left|\Psi_{0}\right\rangle=(|00\rangle+|11\rangle) / \sqrt{2}$ $(\bar{c}(t=0)=1)$ and $\left|\Psi_{0}\right\rangle=\sqrt{1 / 8}|00\rangle+\sqrt{7 / 8}|11\rangle(\bar{c}(t=0) \simeq 0.7)$, under incoherent coupling to a zero temperature environment (i.e., decoherence induced by spontaneous emission). Continuous lines represent exact solutions [19,26,34]; filled squares stem from a randomly chosen unraveling. Symbols show the results for improving unravelings with increasing $\left|\mu_{1}\right|=\left|\mu_{2}\right|=0.8$ (filled diamonds), 1.0 (filled pyramids), 3.0 (filled circles), 4.0 (open squares), 7.0 (open circles), and 15.0 (open diamonds). The dashed line shows the time evolution of $|\Lambda(t)|=\left|\lambda_{1}-\sum_{i=2}^{4} \lambda_{i}\right|$ beyond the disentanglement time $t_{d}$, where the $\lambda_{j}$ are the singular values of the matrix $\left\langle\Psi_{k}^{*}\left|\sigma_{y} \otimes \sigma_{y}\right| \Psi_{i}\right\rangle$ [26], constructed from the pure state decomposition of $\rho(t) .1000$ quantum trajectories were accumulated to generate the unraveling data, in all cases.

with the complex scalar $\mu$, and the left unitary matrix $U$. Now we can minimize $\bar{c}(\delta t)$, by variation of the parameters of the transformation (9), and compare the time evolution under the thus optimized unraveling with the time evolution of concurrence when deduced from the density matrix $\rho(t)$ propagated by the master equation (7). Fig. 6 shows such comparison for two different initial states of two qubits, coupled to a zero temperature environment - i.e., the only source of quantum jumps are spontaneous emission events from either one of the two qubits. For sufficiently large $\mu_{1}=\mu_{2}=\mu \geq 3$ and

$$
U=\left(\begin{array}{cc}
\alpha e^{i \theta} & \beta e^{i \varphi} \\
-\beta e^{-i \varphi} & \alpha e^{-i \theta}
\end{array}\right)
$$

with $\alpha=\beta=1 / \sqrt{2}, \theta+\varphi=\pi / 2=-2 \chi+\sigma, \sigma=\arg \left(\psi_{11}^{2} / r\left(\Psi_{0}\right)\right), r\left(\Psi_{0}\right)=\psi_{00} \psi_{11}-\psi_{01} \psi_{10}$, $\left|\Psi_{0}\right\rangle=\psi_{00}|00\rangle+\psi_{01}|01\rangle+\psi_{10}|10\rangle+\psi_{11}|11\rangle$, and $c\left(\Psi_{0}\right)=2\left|\psi_{01} \psi_{10}-\psi_{00} \psi_{11}\right|$, the agreement is perfect, for all times, despite the fact that we performed the optimization of the jump operators only locally in time, at $t=\delta t$. Note that this is a highly nontrivial result, since, first, not all pure state decompositions of a given density matrix are physically accessible [35] (i.e., in other words, reachable by paramterizations of $L_{k, \pm}$ ), and, second, there is no clear a priori reason why the optimal unraveling should be time-independent, what it is, by virtue of our present results. Note that we obtain qualitatively the same results for different initial states and different types of environment coupling, e.g., dephasing and infinite temperature baths, as well as for tripartite qubit states under zero temperature and dephasing noise [34]. This suggests that the time evolution of entanglement is completely determined by the initial condition and the type of environment coupling, what would imply a considerable simplification of the characterization of mixed state entanglement. Further studies will seek for a mathematical proof of this conjecture, and also for its generalization for higher dimensional bi- or multipartite systems. 

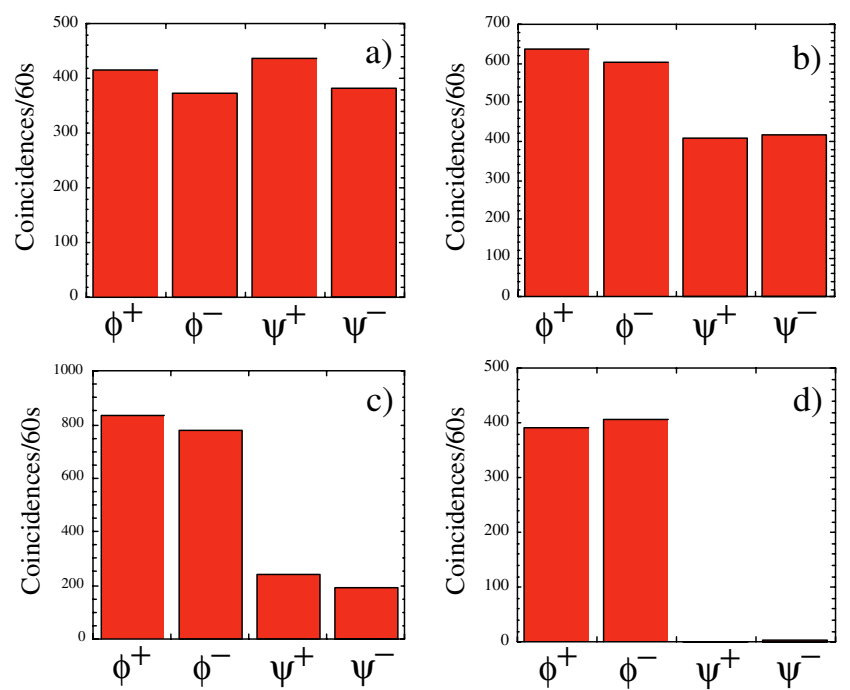

Fig. 7. Relative abundance of detections of the first photon's copies in the symmetric $\left(\psi^{+}, \phi^{ \pm}\right)$ and antisymmetric $\left(\psi^{-}\right)$Bell states, for different initial states $|\Psi\rangle=\alpha|0\rangle \otimes|1\rangle+\beta|1\rangle \otimes|0\rangle$, with $\alpha=0.71 \pm 0.02,0.53 \pm 0.01,0.35 \pm 0.01,0.99 \pm 0.03$, from a) to d) [16].

\section{Observable bipartite entanglement}

Let us finish with a short discussion of yet another strategy for the direct experimental detection of entanglement, which is inspired by the reformulation (4) of pure state concurrence. Obviously, since $A$ is a selfadjoint operator, concurrence can be understood as the expectation value of $A$ with respect to a two-fold copy of the state $|\Psi\rangle$ to be analyzed, and is thus directly accessible through a projective measurement in this extended Hilbert space. Indeed, since $P_{-}^{(1)} \otimes P_{-}^{(2)}+P_{-}^{(1)} \otimes P_{+}^{(2)}=P_{-}^{(1)} \otimes \mathrm{Id}=P_{-}^{(1)} \otimes P_{-}^{(2)}$ on $|\Psi\rangle \otimes|\Psi\rangle$ (since $P_{-}^{(1)} \otimes P_{+}^{(2)}$ is antisymmetric, and therefore vanishes on $|\Psi\rangle \otimes|\Psi\rangle), c(\Psi)$ can be directly measured by projecting either one of the two subsystems together with its copy on the associated antisymmetric subspace, i.e., by evaluating the expectation value of $P_{-}^{(1)} \otimes \operatorname{Id}\left(\right.$ or of $\operatorname{Id} \otimes P_{-}^{(2)}$ ). This has actually been done [16], in a proof of principle experiment on hyperentangled twin photons, where two copies of the same quantum state $|\Psi\rangle=\alpha|0\rangle \otimes|1\rangle+\beta|1\rangle \otimes|0\rangle$ were inscribed in two independent degrees of freedom, the polarization and the momentum, of one and the same physical twin photon pair. The antisymmetric subspace of the first photon and its copy is spanned by the antisymmetric Bell state $\left|\psi^{-}\right\rangle \propto|\rightarrow\rangle \otimes|R\rangle-|\uparrow\rangle \otimes|L\rangle$, and the concurrence of $|\Psi\rangle$ is therefore directly given by the probability to detect the first photon and its copy in the state $\left|\psi^{-}\right\rangle$ (where we identify, without loss of generality, $|\rightarrow\rangle$ and $|L\rangle$ with $|0\rangle$, and $|\uparrow\rangle$ and $|R\rangle$ with $|1\rangle$, respectively). For normalization, also the probability to detect the symmetric Bell states (which complete the four dimensional Bell basis) needs to be recorded in the experiment. This measurement provides an unknown state's $|\Psi\rangle$ concurrence - the experimentalist who performs the projective measurement only needs to be sure that he is given a faithful two-fold copy of $|\Psi\rangle$, but needs no a priori knowledge on $\alpha$ and $\beta[16,36]$. Furthermore, the measurement protocol will succeed for arbitrary pure initial states, not necessarily of the type chosen in this specific experiment, and can be generalized to estimate mixed state entanglement [15].

Fig. 7 shows the relative abundance of antisymmetric and symmetric Bell state detections, for different values of $\alpha$ ( $\beta$ is then fixed by normalization). Fig. 8 collects the resulting values of concurrence, as a function of $|\alpha|$, and shows perfect agreement between experiment and theory. Thus, our algebraic reformulation (4) allowed for the first direct measurement of the entanglement of an unknown pure state, without state tomography. Since (4) is invariant under an increase of the subspaces' dimensions, this paves the way for the direct experimental assessment of the entanglement of unknown quantum states in higher dimensional systems. 


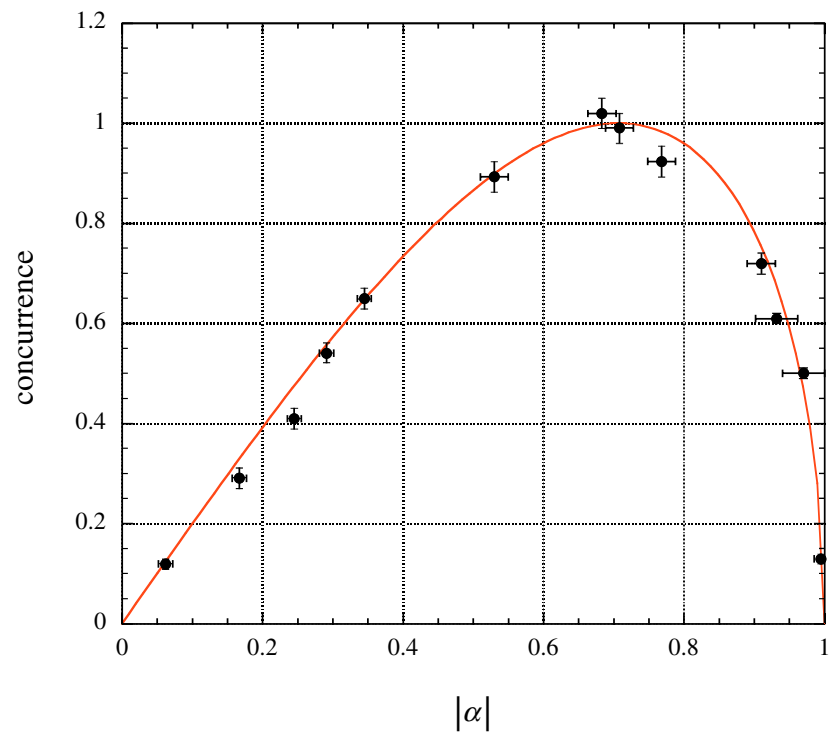

Fig. 8. Experimentally measured concurrence (filled circles) of the state $|\Psi\rangle=\alpha|0\rangle \otimes|1\rangle+\beta|1\rangle \otimes|0\rangle$ vs. $|\alpha|$, compared to the theoretical expectation $c=2|\alpha| \sqrt{1-|\alpha|^{2}}$ (continuous line) [16].

\section{Conclusion}

We introduced various tools and methods for the efficient characterization of quantum entanglement. However, while we initially insisted in the fact that the real challenge lies in the quantitative characterization of the entanglement of higher dimensional bipartite, or multipartite systems, not all of our results do already meet this requirement: The general validity of the unraveling of entanglement also for higher dimensional and/or multipartite systems remains to be shown, and also the direct projective measurement of entanglement on a two-fold copy of the state under scrutiny has hitherto been performed only on pairs of qubits. Hence, lots of hard and challenging work remains to be done, both on the experimental and on the theoretical side: As for the latter, we still need a mathematical proof that all our lower bounds derived from (5) are strictly positive for non-separable states [26], and have to incorporate unavoidably finite detection efficiencies in our unraveling scheme, to make it directly applicable in state of the art experiments.

The results summarized above are the product of a collective effort between Warsaw, Rio de Janeiro, and Dresden. It is a great pleasure to acknowledge the many crucial contributions due to Olivier Brodier, Marc Busse, Luiz Davidovich, Rafał Demkowicz-Dobrzański, Ignacio Garcia-Mata, Marek Kuś, Paulo Souto Ribeiro, Carlos Viviescas, and Steven Walborn. The collaboration between Dresden and Warsaw was funded by VolkswagenStiftung, the one between Dresden and Rio by the DAAD, within a PostDoc fellowship (F.M.), and the PROBRAL program.

\section{References}

1. D. Bouwmeester, J.W. Pan, K. Mattle, M. Eibl, H. Weinfurter, A. Zeilinger, Nature 390, 575 (1997)

2. R. Raussendorf, H.-J. Briegel, Phys. Rev. Lett. 86, 5188 (2001)

3. R.F. Werner, Phys. Rev. A 40, 4277 (1989)

4. I. Cirac, P. Zoller, Phys. Rev. Lett. 74, 4091 (1995)

5. F. Schmidt-Kaler, H. Häffner, M. Riebe, S. Gulde, G.P.T. Lancaster, T. Deuschle, C. Becher, C.F. Roos, J. Eschner, R. Blatt, Nature 422, 408 (2003)

6. H. Häffner, W. Hänsel, C.F. Roos, J. Benhelm, D. Chek-al-kar, M. Chwalla, T. Körber, U.D. Rapol, M. Riebe, P.O. Schmidt, C. Becher, O. Gühne, W. Dür, R. Blatt, Nature 438, 643 (2005) 
7. D. Leibfried, E. Knill, S. Seidelin, J. Britton, R.B. Blakestad, J. Chiaverini, D.B. Hume, W.M. Itano, J.D. Jost, C. Langer, R. Ozeri, R. Reichle, D.J. Wineland, Nature 438, 639 (2005)

8. C.-Y. Lu, X.-Q. Zhou, O. Gühne, W.-B. Gao, J. Zhang, Z.-S. Yuan, A. Goebel, T. Yang, J.-W. Pan, Nat. Phys. 3, 91 (2007)

9. R. Alicki, Chem. Phys. 322, 75 (2006)

10. A.R.R. Carvalho, F. Mintert, A. Buchleitner, Phys. Rev. Lett. 93, 230501 (2004)

11. C. Simon, J. Kempe, Phys. Rev. A 65, 052327 (2002)

12. W. Dür, H.-J. Briegel, Phys. Rev. Lett. 92, 180403 (2004)

13. O. Gühne, M. Reimpell, R.F. Werner, Phys. Rev. Lett. 98, 110502 (2007)

14. N. Kiesel, C. Schmid, U. Weber, O. Gühne, G. Tóth, R. Ursin, H. Weinfurter, Phys. Rev. Lett. 95, $210502(2005)$

15. F. Mintert, A. Buchleitner, An observable entanglement measure for unknown mixed quantum states (2007) [quant-ph/0605250]

16. S.P. Walborn, P.H. Souto Ribeiro, L. Davidovich, F. Mintert, A. Buchleitner, Nature 440, 1022 (2006)

17. M. Brune, E. Hagley, J. Dreyer, X. Maître, A. Maali, C. Wunderlich, J.M. Raimond, S. Haroche, Phys. Rev. Lett. 77, 4887 (1996)

18. M. Arndt, K. Hornberger, A. Zeilinger, Phys. World 18, 35 (2005)

19. W.K. Wootters, Phys. Rev. Lett. 80, 2245 (1998)

20. C.F. Roos, G.P.T. Lancaster, M. Riebe, H. Häffner, W. Hänsel, S. Gulde, C. Becher, J. Eschner, F. Schmidt-Kaler, R. Blatt, Phys. Rev. Lett. 92, 220402 (2004)

21. G. Puentes, A. Aiello, D. Voigt, J.P. Woerdman, Phys. Rev. A 75, 032319 (2007)

22. M.P. Almeida, F. de Melo, M. Hor-Meyll, A. Salles, S.P. Walborn, P.H. Souto Ribeiro, L. Davidovich, Experimental observation of environment-induced sudden death of entanglement (2007) [quant-ph/0701184]

23. M.A. Nielsen, I.L. Chuang, Quantum Computation and Quantum Information (Cambridge University Press, Cambridge, 2000)

24. P. Rungta, V. Buzek, C.M. Caves, M. Hillery, G.J. Milburn, Phys. Rev. A 64, 042315 (2001)

25. A. Uhlmann, Phys. Rev. A 62, 032307 (2000)

26. F. Mintert, A.R.R. Carvalho, M. Kuś, A. Buchleitner, Phys. Rep. 415, 207 (2005)

27. F. Mintert, M. Kuś, A. Buchleitner, Phys. Rev. Lett. 95, 260502 (2005)

28. R. Demkowicz-Dobrzański, A. Buchleitner, M. Kuś, F. Mintert, Phys. Rev. A 74, 052303 (2006)

29. F. Mintert, M. Kuś, A. Buchleitner, Phys. Rev. Lett. 92, 167902 (2004)

30. F. Mintert, A. Buchleitner, Phys. Rev. A 72, 012336 (2005)

31. A.R.R. Carvalho, F. Mintert, S. Palzer, A. Buchleitner, Eur. Phys. J. D 41, 425 (2007)

32. I. García-Mata, A.R.R. Carvalho, F. Mintert, A. Buchleitner, Phys. Rev. Lett. 98, 120504 (2007)

33. H. Carmichael, An Open Systems Approach to Quantum Optics, Lecture Notes in Physics (SpringerVerlag, Berlin, 1993)

34. A.R.R. Carvalho, M. Busse, O. Brodier, C. Viviescas, A. Buchleitner, Unravelling entanglement (2005) [quant-ph/0510006]

35. H.M. Wiseman, J.A. Vacaro, Phys. Rev. Lett. 87, 240402 (2001)

36. S. van Enk, Can measuring entanglement be easy? (2006) [quant-ph/0606017] 